\title{
Computed tomography of pulmonary sarcoid-like granulomas induced by complete Freund's adjuvant in rats
}

\author{
A. Bergeron*, J-P. Laissy\#, P. Loiseau*, E. Schouman-Claeys", A.J. Hance*, A. Tazi*,
}

Computed tomography of pulmonary sarcoid-like granulomas induced by complete Freund's adjuvant in rats. A. Bergeron, J-P. Laissy, P. Loiseau, E. Schouman-Claeys, A.J. Hance, A. Tazi. C ERS Journals Ltd 2001.

ABSTRACT: Intravenous injection of complete Freund's adjuvant (CFA) in rats has been proposed as an experimental model for pulmonary sarcoidosis, but only some animals develop granulomas. Because the detection of the presence of granulomas and evaluation of the extent of the reaction has required histological evaluation, this model has been of limited use in following the evolution of the granulomatous process.

The present study evaluated the ability of lung computed tomography (CT) scanning to identify in vivo pulmonary granulomas induced by CFA in rats. Wistar rats were injected with CFA to induce pulmonary sarcoid-like granulomas, and the presence and extent of pulmonary abnormalities, as detected by spiral CT and histopathological analysis, were compared.

Spiral CT had a high sensitivity and specificity for the detection of sarcoid-like granulomas in rats injected with CFA. The extent of the pulmonary granulomatous reaction as assessed by the two techniques strongly correlated $(r=0.93, p<0.01)$. In contrast, the mean density of lungs containing granulomas was not significantly different from that of lungs with no granulomatous reaction.

Thus, lung computed tomography appears to be a valuable tool for the in vivo evaluation of the pulmonary granulomatous reaction induced by complete Freund's adjuvant in rats. With the help of computed tomography, this experimental model should be suitable for the sequential study of pulmonary sarcoid-like granulomas, particularly in response to various therapeutic strategies.

Eur Respir J 2001; 18: 357-361.
*INSERM U82, Faculté de Médecine Xavier Bichat, Paris, France, ${ }^{\#}$ Laboratoire d'Imagerie Médicale Paris-Nord, Faculté de Médecine Xavier Bichat, Paris, France and "Service de Pneumologie, Hôpital Avicenne, Bobigny, France.

Correspondence: A. Tazi, Service de Pneumologie, Hôpital Avicenne, 125, rue de Stalingrad, 93009 Bobigny, France.

Fax: 33148955126

Keywords: Computed tomography scan

experimental model

granulomas

sarcoidosis

Received: October 22000

Accepted after revision March 292001
Pulmonary involvement is one of the most common features of sarcoidosis and can lead to severe pulmonary fibrosis in some patients $[1,2]$. Considerable effort has been made to better understand the steps involved in the development and resolution of immune granulomas, the structures responsible for the destruction of normal lung tissue in this disease [3-5]. The lack of an appropriate experimental model for sarcoidosis, however, has hampered the sequential study of the granulomatous reaction, and currently, little is known concerning the factors that determine whether granulomatous lesions progress or heal spontaneously $[3,4]$.

Intravenous injection of complete Freund's adjuvant (CFA) in rats has been found to induce sarcoid-like reactions [6]. Noncaseating granulomas containing epithelioid and giant cells surrounded by lymphocytes, which closely approximate those observed in human pulmonary sarcoidosis, are observed in subpleural and peribronchovascular areas of the lung of injected animals [6]. For unknown reasons, however, the extent of the pulmonary granulomatous reaction has been found to be quite variable, even when congenic animals are evaluated in the same experiment, and some animals develop only moderate cellular infiltration without granuloma formation [6]. Because it was not possible to predict which animals had developed granulomas without sacrificing the animals and examining the lung parenchyma, this model has had major limitations in studies designed to evaluate the evolution of the granulomatous process, particularly in response to various treatments.

Computed tomography (CT) of the lungs is a very sensitive method for detecting the lesions of pulmonary sarcoidosis $[2,7-10]$. The most frequent features observed are irregular thickening of bronchovascular bundles and small nodules distributed along vessels and under the pleura [2, 7-10]. Correlations with lung histopathology have demonstrated that these radiological abnormalities correspond to granulomas present in the connective tissue sheaths surrounding pulmonary vessels and airways and in the subpleural regions of the lung [11]. In this context, the purpose of the present study was to evaluate the ability of CT to detect the development of pulmonary sarcoid-like granulomas in rats injected with CFA. To do so, the results of lung CT were correlated with pulmonary histopathological features of the same animals. 


\section{Materials and methods}

Eight male Wistar rats (300-350 g, Charles River, St Aubin les Elbeuf, France) were provided food and water ad libitum. To initiate studies, $0.20 \mathrm{~mL}$ of CFA (Difco, Detroit, USA) was injected into the dorsal tail vein on two successive days. Following these injections, once daily subcutaneous injections of vancomycin (Lilly, St Cloud, France) were administered for 5 days $\left(20 \mathrm{mg} \cdot \mathrm{kg}^{-1} \cdot \mathrm{day}^{-1}\right)$. The experimental protocol was approved by the University Animal Care and Use Committee.

\section{Evaluation of lung involvement by computed tomo- graphy}

Preliminary time course experiments have shown that pulmonary sarcoid-like granulomas were well developed 28 days after the injections of CFA, and that the lesions were still florid on day 58. For practical reasons, CT was performed 33 days after the first injection of CFA.

To perform $\mathrm{CT}$, rats were anaesthetized in a cylindrical tube (1 L volume) by flushing the chamber continuously at $5 \mathrm{~L} \cdot \mathrm{min}^{-1}$ with $1 \%$ Isoflurane (Abbot, Queenborough, UK)/99\% air. Following the procedure (duration $\sim 10 \mathrm{~min}$ ) all animals aroused spontaneously. The rats were not intubated and the animals were evaluated while breathing spontaneously. Helical acquisitions were obtained using a CT-Twin Scanner (Picker-Elscint, Haifa, Israel). This dual-slice helical scanner permits the same volume of tissue to be acquired in half the time required by a single-slice scanner. Acquisitions were obtained from the lung apices to the diaphragm as follows: collimation of $1.1 \mathrm{~mm}$, pitch of 1.5 (table-top speed of $1.5 \mathrm{~mm} \cdot \mathrm{s}^{-1}$ ), $120 \mathrm{kV}$ and $266 \mathrm{mAs}, 1-\mathrm{s}$ scan time, scan angle of $360^{\circ}$. The field of view was $180 \mathrm{~mm}$, and the matrix was $512 \times 512$, resulting in a resolution of $0.35 \times 0.35 \times 1 \mathrm{~mm}$ per voxel with acceptable signalto-noise ratio. For optimal nodule visualization, images were reconstructed using a high spatial frequency kernel and a 180 degree linear interpolation algorithm. Helical images were reconstructed at $1 \mathrm{~mm}$ intervals. The images were obtained at standard window levels and widths for lung parenchyma (level=-600 HU, width=1,600 HU). The CT scans were reviewed by three observers unaware of pathological findings and conclusions were reached by consensus. Scans were assessed for the presence and distribution of parenchymal nodules and the size of the nodules (maximal diameter of the largest nodules) as determined by caliper measurements on-screen or manual measurements on film. To evaluate the extent of lung abnormalities, both lungs were divided into three areas (upper, middle and lower zone) and a score was established based on the absence $(0)$, or presence (1) of nodules on each of the six lung CT slices analysed for a given animal. Thus, for each rat, the score could vary from 0 (no nodules on the six lung CT slices analysed) to 6 (presence of nodules on all six lung CT slices). Lung involvement was considered as patchy (score 1-3) or diffuse (score 4-6). The mean lung density was also determined as previously described [12]. Briefly, the entire right and left lungs were outlined separately and the average CT attenuation values were recorded for each region.

\section{Processing of the lungs and morphological assessment}

All rats were sacrificed immediately after CT scanning (mean \pm SD weight $437.9 \pm 14.9 \mathrm{~g}$ ). After receiving an intraperitoneal injection of $0.4 \mathrm{~mL}$ of pentobarbital (Sanofi, Libourne, France) per $300 \mathrm{~g}$ of body weight, anaesthetized animals were exsanguinated. The anatomy of the right and left lungs of the rat are different: the right lung is composed of three main lobes (superior, middle and inferior) and one minor lobe lying above the diaphragm, whereas the left lung has a single lobe. The three major right lobes were separated and the left lung was divided into three pieces of approximately equivalent size. The diaphragmatic lobe was not studied because of its small size. Lung fragments were immediately frozen and stored in liquid nitrogen until use for morphological assessment. Histopathological features of the frozen lungs were evaluated using $4 \mathrm{~mm}$ cryostat sections of each lobe stained with haematoxylin and eosin. The lobes were sliced in the transverse plan to correspond as closely as possible with the CT slices. Lung sections and CT images were examined in a blind fashion. All the tissue sections were examined for each animal. A histological score was defined according to the presence (1) or absence (0) of granulomas on each of the six lung tissue sections examined. For each rat, the score could vary from 0 (no granuloma in any tissue section) to 6 (presence of granulomas in all the six lung sections). The granulomatous reaction was graded as follows: no granulomas, localized reaction (score 1-3), diffuse reaction (score 4-6). Complete agreement in scoring was obtained between two independent observers.

\section{Statistical analysis}

All data are expressed as mean \pm SD. The sensitivity and specificity of spiral acquisitions to detect pulmonary sarcoid-like granulomas were evaluated by comparing the results to those obtained by histological evaluation. The extent of the pulmonary granulomatous reaction was correlated to the extent of lung abnormalities on CT scan using $\kappa$ statistics (1-0.81 excellent; $0.8-0.61$ good; $0.6-0.41$ moderate; 0.4-0.21 poor; $<0.2$ bad) [13]. Quantitative radiological and histological scores were also correlated using Spearman's test; a $\mathrm{p}<0.05$ was considered significant.

\section{Results}

Detection by computed tomography scan of sarcoidlike pulmonary granulomas in rats injected with complete Freund's adjuvant

Lung CT demonstrated the presence of subpleural or peribronchovascular nodules in $6 / 8$ of the animals 
examined. These nodules ranged in size from 0.4 $0.8 \mathrm{~mm}$ in diameter (fig. 1). The distribution of pulmonary nodules was similar comparing the upper and lower parts of the lungs, but they were more frequently observed in the right lung.

Histological evaluation of lung tissue also demonstrated the presence of granulomas in the lungs of $6 / 8$ rats. These lesions were very similar to those observed in the course of human sarcoidosis. The granulomas were composed of a central core of macrophages, epithelioid cells and giant cells surrounded by lymphocytes, and they were essentially only found in subpleural and peribronchovascular regions (fig. 1). The size of lung granulomas varied from $0.1-0.5 \mathrm{~mm}$ diameter. In all cases, granulomas of different sizes were observed at different sites in the lungs of a particular rat.

Results of CT scans were in concordance with those of lung histopathology. In particular, no pulmonary nodules were detected by CT in the lungs of rats that were found not to contain granulomas after extensive microscopic examination of lung tissue. Thus, the sensitivity and the specificity of CT for the detection of the presence of lung granulomas were both $100 \%$. In contrast, measurement of mean lung density was not different comparing rats that developed pulmonary sarcoid-like granulomas and those which did not $(-563 \pm 12 \mathrm{HU}$ and $-567 \pm 72 \mathrm{HU}$, respectively).

Accuracy of lung computed tomography scan to evaluate the extent of the granulomatous reaction in rats injected with complete Freund's adjuvant

It can be useful to evaluate the extent of the granulomatous reaction sequentially in the same animal in response to various therapeutic strategies. Therefore, it was important to determine whether the results obtained by CT reliably reflected the extent of lung infiltration by the granulomatous reaction. To do
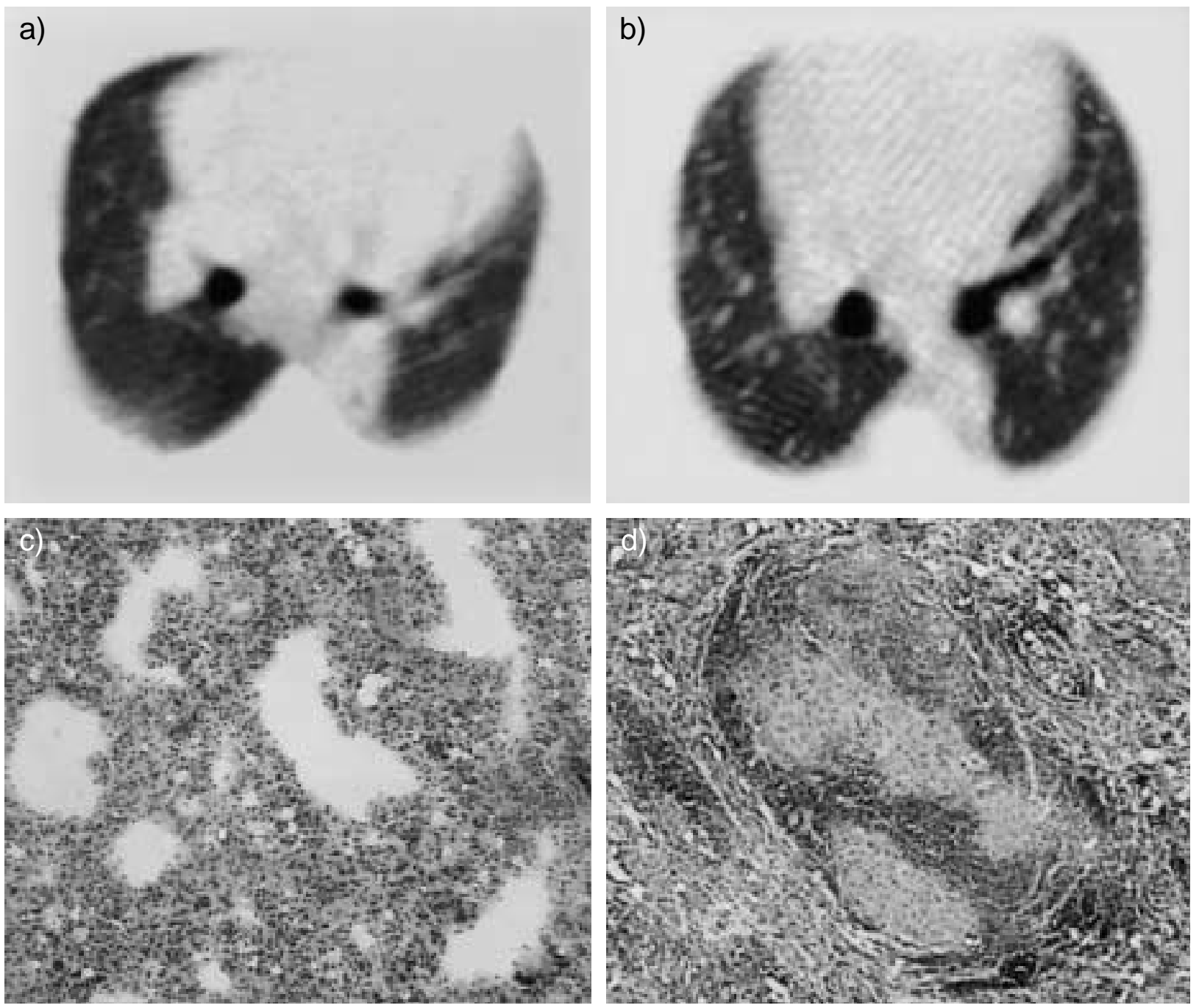

Fig. 1.-Evaluation by dual helical lung computed tomography (CT) of pulmonary sarcoid-like granulomas induced by complete Freund's adjuvant (CFA) in rats. Lung CT from two rats injected with CFA: a) normal lung CT and b) presence of pulmonary nodules. Photomicrographs of lung parenchyma from the same rats stained with haematoxylin and eosin: c) moderate cellular infiltration without granulomas and d) typical sarcoid-like granulomas. The lungs were not inflated prior to fixation, and atelectatic areas are present. Original magnification $\times 125$. 
so, the extent of pulmonary nodular infiltration as assessed by CT and histopathological examination were compared. At light microscopy, the granulomatous response was absent in two animals, localized in three animals and diffuse in the three others; whereas, pulmonary nodular infiltration on CT scans was absent in two animals, patchy in four rats and diffuse in the remaining two (table $1, \kappa=0.82$ ). Furthermore, despite the small number of animals studied, a correlation was observed between the radiological and histological scores evaluating the profusion of changes in the rats' lungs as assessed respectively by $\mathrm{CT}$ and light microscopy $(\mathrm{r}=0.93, \mathrm{p}<0.01)$. In contrast, mean lung density was not correlated with the extent of the granulomatous reaction as assessed by histopathology (no granulomas: $-563 \pm 12 \mathrm{HU}$; localized granulomatous lung infiltration: $-614 \pm 58 \mathrm{HU}$; diffuse granulomas: $-521 \pm 52 \mathrm{HU}$ ).

\section{Discussion}

In this preliminary study, it has been shown that: 1) dual-helical CT was highly sensitive and specific for the detection of sarcoid-like granulomas in the lungs of rats injected with CFA; 2) there was a tight correlation between light microscopy and $\mathrm{CT}$ for the evaluation of the extent of the granulomatous reaction in the lungs of these animals; and 3) the global measurement of mean lung density was not useful for the evaluation of pulmonary granulomas.

Few reports are available concerning the use of pulmonary CT in animals. Most of these studies have used high-resolution CT to evaluate pigs in different pathological situations [14-17]. In this setting, the radiological abnormalities observed correlated well with the underlying histopathological features present in the lungs [14-17]. Less data are available concerning the use of lung CT in smaller animals, for which spatial and contrast resolution are limited. Lung CT, however, has been performed in rats for the evaluation of radiation-induced lung injury, but only lung density was considered, and radiological patterns were not precisely described [12, 18, 19]. In the present study, it was demonstrated that dual-helical lung CT is efficient for the detection of pulmonary sarcoid-like granulomas in the rats injected with CFA. The technique used here showed the presence of pulmonary nodules in all animals that had pulmonary

Table 1.-Correlation between results of lung computed tomography (CT) scans and histological evaluation for the assessment of the extent of the pulmonary granulomatous response induced by complete Freund's adjuvant in rats*

\begin{tabular}{lccc}
\hline Nodules on lung & \multicolumn{3}{c}{$\begin{array}{c}\text { Pulmonary granulomas at } \\
\text { light microscopy }\end{array}$} \\
\cline { 2 - 4 } & Absent & Localized & Diffuse \\
\hline Absent & 2 & 0 & 0 \\
Patchy & 0 & 3 & 1 \\
Diffuse & 0 & 0 & 2 \\
\hline
\end{tabular}

*: $\kappa=0.82$ for the concordance between the two methods. granulomas at the light microscopic level and it was also specific. Furthermore, although the number of animals studied was small, the results also suggest that data obtained by CT accurately reflect the extent of pulmonary granulomatous reaction induced by CFA.

Despite the high sensitivity of the CT scan technique used for the detection of pulmonary sarcoid-like granulomas in the present study, some limitations were encountered for the precise description of lung involvement. One limitation was the blurring of nodules because of respiratory and cardiac motion artefacts. Respiratory motion artefacts were, however, attenuated by the dual-helix. Artefacts due to respiration have previously been found not to significantly modify the detection of lung metastases in dogs using spiral CT [20]. Acquisition during spontaneous respiration can also produce patchy areas of apparently increased density. Thus, it was not possible to quantify "ground-glass" opacities resulting from areas of inflammation associated with the granulomatous response.

It has previously been shown that cardiac motion artefacts on spiral CT affect almost exclusively the lingula and lower lobe segments, those in close contact with the left ventricle [21]. This caveat was not important here because granulomas were not localized to a small region of the lungs, thereby permitting the evaluation of regions undisturbed by cardiac motion.

Another limitation was the difficulty in determining the precise anatomical localization of the nodules detected by CT. Localization is greatly facilitated by the identification of lung fissures. In the rat lung, however, the left lung is not segmented by fissures, and the fissures of the right lung were not always seen on $\mathrm{CT}$, thus depriving the reader of these anatomical markers.

Measurement of the mean lung density has previously been used to study radiation induced lung injury in rats [12, 18, 19]. This index, however, is relatively crude and results can be influenced by many factors, including the lung area scanned and the age of the animal [22]. Here, it was found that mean lung density was not a good parameter to distinguish rats that developed lung granulomas in response to CFA from those that did not. The acquisition of the scans during spontaneous respiration might have introduced some bias in the measurement of lung density. Alternatively, it is possible that even when pulmonary granulomas are present diffusely, the overall surface of the lungs occupied by the small nodules is not sufficient to modify the mean lung density.

In summary, results of this preliminary study clearly demonstrate that it is possible to analyse pulmonary parenchyma of rats with computed tomography and to detect pulmonary nodules by dual helical computed tomography in these small animals. This technique should be useful in the evaluation of experimental models of lung diseases that result in the formation of discrete parenchymal lesions. In particular, the presented findings suggest that it may be useful to sequentially study animals with established sarcoid-like granulomas, thereby permitting the evaluation of the effects of various potential therapeutic strategies. The identification of 
therapies that accelerate the resolution of established granulomatous lesions could prove useful in the treatment of patients with sarcoidosis.

Acknowledgements. The authors thank M-C. Pruvost for her excellent technical assistance.

\section{References}

1. Scadding JG, Mitchell DN. Sarcoidosis. 2nd Edn. London, Chapman Hall, 1985.

2. Statement on sarcoidosis. Joint statement of the American Thoracic Society (ATS), the European Respiratory Society (ERS) and the World Association of Sarcoidosis and other Granulomatous Disorders (WASOG) adopted by the ATS Board of Directors and by the ERS Executive Committee, February 1999. Am J Respir Crit Care Med 1999; 160: 736-755.

3. Robinson DS, Richeldi L, Saltini C, du Bois RM. Granulomatous processes. In: Crystal RG, West JB, Weibel ER, Barnes PJ, eds. The Lung. Scientific Foundations. 2nd Edn. Philadelphia, LippincottRaven, 1997; 2395-2409.

4. Agostini C, Adami F, Semenzato G. New pathogenetic insights into the sarcoid granuloma. Curr Opin Rheumatol 2000; 12: 71-76.

5. Bergeron A, Bonay $\mathrm{M}$, Kambouchner $\mathrm{M}$, et al. Cytokine patterns in tuberculous and sarcoid granulomas: correlations with histopathologic features of the granulomatous response. J Immunol 1997; 159: 3034-3043.

6. Soler P, Bernaudin JF, Basset F. Ultrastructure of pulmonary granulomatosis induced in rats by intravenous complete Freund's adjuvant. Virchows Arch A Path Anat and Histol 1975; 368: 35-50.

7. Brauner MW, Grenier P, Mompoint D, Lenoir S, de Cremoux H. Pulmonary sarcoidosis: evaluation with high-resolution CT. Radiology 1989; 172: 467-471.

8. Muller NL, Kullnig P, Miller RR. The CT findings of pulmonary sarcoidosis: analysis of 25 patients. $A J R$ 1989; 152: 1179-1182.

9. Abehsera M, Valeyre D, Grenier P, Jaillet H, Battesti JP, Brauner MW. Sarcoidosis with pulmonary fibrosis: CT patterns and correlation with pulmonary function. AJR 2000; 174: 1751-1757.

10. Haramati LB, Lee G, Singh A, Molina PL, White CS.
Newly diagnosed pulmonary sarcoidosis in HIVinfected patients. Radiology 2001; 218: 242-246.

11. Nishimura $\mathrm{K}$, Itoh $\mathrm{H}$, Kitaichi $\mathrm{M}$, Nagai $\mathrm{S}$, Izumi $\mathrm{T}$. Pulmonary sarcoidosis: correlation of CT and histopathologic findings. Radiology 1993; 189: 105-109.

12. el-Khatib E, Lehnert S. Lung density changes observed in vivo in rat lungs after irradiation: variations among and within individual lungs. Int $J$ Radiat Oncol Biol Phys 1989; 16: 745-754.

13. Landis JR, Koch GG. The measurement of observer agreement for categorical data. Biometrics 1977; 33: 159-174.

14. Noma S, Herman PG, Khan A, Rojas KA, Pipman Y. Sequential morphologic changes of elastase-induced pulmonary emphysema in pig lungs. Evaluation by high-resolution computed tomography. Invest Radiol 1991; 26: 446-453.

15. Horvath G, Noma S, Moskowitz GW, Khan A, Balazs G, Herman PG. Experimental pulmonary infarction in a pig model. Invest Radiol 1992; 27: 829-835.

16. Balazs G, Noma S, Khan A, Eacobacci T, Herman PG. Bleomycin-induced fibrosis in pigs: evaluation with CT. Radiology 1994; 191: 269-272.

17. Takahashi M, Balazs G, Pipman Y, et al. Radiationinduced lung injury using a pig model. Evaluation by high-resolution computed tomography. Invest Radiol 1995; 30: 79-86.

18. Ward WF, Lin PJ, Wong PS, Behnia R, Jalali N. Radiation pneumonitis in rats and its modification by the angiotensin-converting enzyme inhibitor captopril evaluated by high-resolution computed tomography. Radiat Res 1993; 135: 81-87.

19. Vujaskovic Z, Down JD, van $\mathrm{t}^{\prime}$ Veld AA, et al. Radiological and functional assessment of radiationinduced lung injury in the rat. Exp Lung Res 1998; 24: $137-148$

20. Coakley FV, Cohen MD, Waters DJ, et al. Detection of pulmonary metastases with pathological correlation: effect of breathing on the accuracy of spiral CT. Pediair Radiol 1997; 27: 576-579.

21. Rémy-Jardin M, Rémy J, Giraud F, Marquette $\mathrm{CH}$. Pulmonary nodules: detection with thick-section spiral CT versus conventional CT. Radiology 1993; 187: 513520.

22. Lehnert S, Schreiner LJ, el-Khatib E. Factors influencing lung density in experimental models: results of studies using CT densitometry. Physiol Meas 1993; 14: 183-193. 